\section{Big MAC attack in osteoarthritis}

\section{By Michael J. Haas, Senior Writer}

An international research team has shown that inhibiting the complement system slows disease progression in mice with osteoarthritis, thus pointing to complement inhibitors as potential disease modifiers. ${ }^{1}$ Although the findings offer companies developing inhibitors of the complement system a potential new indication, the safety and side effects of long-term inhibition will have to be determined first.

There are several approved osteoarthritis (OA) therapies on the market that treat disease-related pain and inflammation, but none targets the underlying processes that lead to cartilage destruction and bone spurs in the joints.

Multiple studies have implicated the complement system in degenerative diseases such as age-related macular degeneration $(\mathrm{AMD})^{2-4}$ and Alzheimer's disease (AD).$^{5-7}$ Thus, a team led by William Robinson wanted to know whether complement also played a role in joint degeneration in OA.

Robinson is associate professor of medicine in the division of immunology and rheumatology at Stanford University School of Medicine and a staff physician in the VA Palo Alto Health Care System.

The group found that synovial fluid and synovial membranes from OA patients had higher levels of complement system components than fluid and membranes from healthy controls. These components included complement 5 (C5) and the complement C5b-9 membrane attack complex (MAC). C5 plays a role in inflammatory and cell-killing processes by cleaving into $\mathrm{C} 5 \mathrm{a}$ and C5b, respectively, whereas MAC creates a transmembrane channel on the surface of target cells and destroys them.

In a mouse model of knee surgery-induced OA, animals deficient in C5 had less cartilage damage, inflammation of the synovium and bone spur formation than wild-type models. Also, wild-type models treated with an anti-C5 antibody or a C5-inhibiting fusion protein developed less severe disease than wild-type models that received inactive controls.

Models deficient in the MAC inhibitor Cd59 had greater disease severity than wild-type controls. Similar results were seen in a medial meniscal tearing model of $\mathrm{OA}$.

Lastly, the team showed that MAC formation in normal human chondrocyte cultures was induced by fibromodulin and aggrecan, which are two components of cartilage extracellular matrix (ECM) that can enter the synovium following cartilage damage. In turn, MAC induced the chondrocytes to produce cartilage-degrading enzymes, C5, C3 and proinflammatory proteins.

In its report in Nature Medicine, the team wrote that its findings suggest joint damage can release ECM components into the synovium to trigger an ongoing cycle of complement activation and MAC-induced cartilage degradation that promotes OA (see Figure 1, "Complement's vicious cycle in osteoarthritis").

Thus, blocking complement activation during the early stages of disease could decrease or even prevent joint degeneration, Robinson told SciBX.

The team included researchers from Brigham and Women's Hospital, Harvard Medical School, the Case Western Reserve University School of Medicine, the Hospital for Special Surgery, Rush University Medical Center, the University of Colorado Denver's Health Sciences Center, the University of Padova and Novartis AG's Novartis Institutes for BioMedical Research, which conducted the analyses on the human synovial samples.

"The team's animal studies clearly show that C5 is one of the key factors in the pathogenesis of osteoarthritis and that C5 inhibitors could be used to treat it," said Seonggu Ro, EVP and CTO of CrystalGenomics Inc.

"This is a potentially groundbreaking publication if its findings can be translated into humans. C5 inhibition could certainly be a way to treat osteoarthritis, and it should definitely be explored clinically," said Axel Vater, VP and head of drug discovery at Noxxon Pharma AG.

CrystalGenomics' CG100649, a dual-acting cyclooxygenase-2 (COX-2) and carbonic anhydrase inhibitor, is in Phase IIb testing to treat OA.

Noxxon's NOX-D15, an L-aptamer that inhibits $\mathrm{C} 5 \mathrm{a}$, is in discovery research to treat AMD.

\section{Local strategies}

Because C5 is involved in several immune responses, both Ro and Vater said systemic inhibition of the target could have serious side effects. For example, the one approved C5 inhibitor-Soliris eculizumab from Alexion Pharmaceuticals Inc. - carries a black box warning of an increased risk of meningococcal infections.

Soliris is approved to treat atypical hemolytic uremic syndrome (aHUS) and paroxysmal nocturnal hemoglobinuria (PNH) and is in Phase II testing to treat multiple hematological, neurological and renal indications.

Ro, Vater and Robinson thought local (intra-articular) inhibition of C5 or other complement factors would likely be a better option in OA than systemic inhibition.

Ro added that safety would be particularly critical if chronic C5 inhibition were required to prevent OA. "If the inhibitor had even minor side effects, physicians would be very reluctant to prescribe it" as a prophylactic in otherwise healthy individuals, he said.

Therefore, Vater said he would be "interested to see further studies that examine whether a single prophylactic treatment right after surgery or trauma would be sufficient to prevent osteoarthritis, or whether complement needs to be inhibited chronically" to prevent post-surgical OA. 


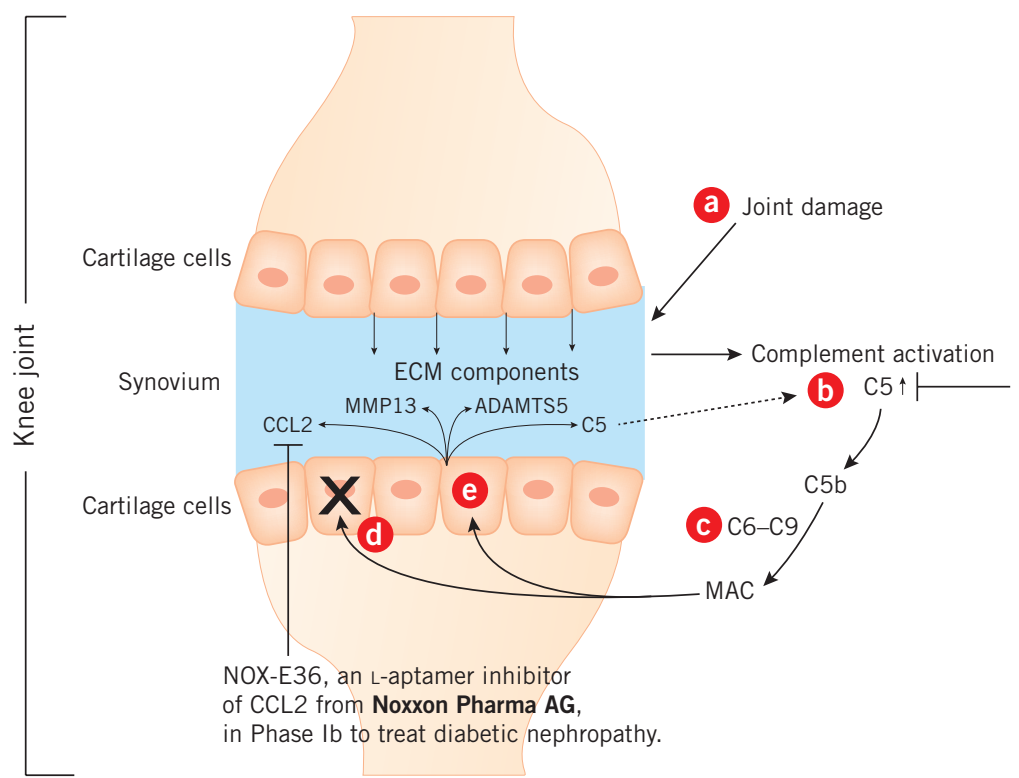

Soliris eculizumab, a humanized $m A b$ targeting C5 from Alexion Pharmaceuticals Inc. (NASDAQ:ALXN), approved to treat atypical hemolytic uremic syndrome (aHUS) and paroxysmal nocturnal hemoglobinuria (PNH) and in Phase II to treat multiple hematological, neurological and renal indications. ARC1905, an anti-C5 aptamer from Archemix Corp. and Ophthotech Corp. in Phase I to treat age-related macular degeneration (AMD).

Figure 1. Complement's vicious cycle in osteoarthritis. Joint damage can activate a self-perpetuating cycle of complement activation and cartilage damage that leads to osteoarthritis.

Joint damage from injury or surgery releases extracellular matrix (ECM) components [a], such as fibromodulin and aggrecan, into the synovial fluid that activate the complement system and upregulate complement 5 (C5) [b]. Upon cleavage from C5, C5b recruits other complement factors (C6-C9) to form the complement C5b-9 membrane attack complex (MAC) [c] that targets chondrocytes and either kills them to cause cartilage damage [d] or induces expression of proinflammatory monocyte chemoattractant protein-1 (MCP-1; CCL2), expression of additional C5 and secretion of ECM-degrading enzymes such as matrix metalloproteinase 13 (MMP13) and ADAM metallopeptidase with thrombospondin type 1 motif 5 (ADAMTS5) [e], thereby continuing the cartilage-damaging cycle [a-e].

Ergidina, a recombinant human minibody against C5 fused with an arginine-glycine-aspartic acid motif, from Adienne Pharma \& Biotech, is in preclinical testing to treat ischemia/reperfusion injury.

He noted that in the team's surgically induced models of OA, the lag time between knee surgery and the onset of cartilage damage was 12 weeks. A lag time is also observed in humans following knee injury or trauma, he said.

Robinson thought a brief period of complement inhibition would be sufficient to break the cycle of complement activation and cartilage damage and thus prevent osteoarthritis after knee trauma. He said his team is now investigating that preventive strategy in both the surgical and meniscal tearing mouse models of $\mathrm{OA}$.

Vater also said that the C5a inhibitor NOX-D15 would probably have no therapeutic effect in OA because the leukocyte infiltration induced by $\mathrm{C} 5 \mathrm{a}$ is not found in osteoarthritic joints and the paper showed no role for $\mathrm{C} 5 \mathrm{a}$ in the disease.

However, he noted that the team's findings in human chondrocytes showed that MAC upregulated the proinflammatory protein monocyte chemoattractant protein-1 (MCP-1; CCL2). "It may be worthwhile to test our CCL2 inhibitor, NOX-E36, in a model of osteoarthritis," he said.

NOX-E36 is in Phase Ib testing to treat diabetic nephropathy.

In addition to the prophylactic studies in mice, Robinson said his team's ongoing work includes investigating how the complement system becomes activated in $\mathrm{OA}$ and how best to block that activation.

Stanford University has filed a patent application covering the findings, and the IP is available for licensing.

Haas, M.J. SciBX 4(47); doi:10.1038/scibx.2011.1311

Published online Dec. 8, 2011

\section{REFERENCES}

1. Wang, Q. et al. Nat. Med.; published online Nov. 6, 2011; doi:10.1038/nm.2543

Contact: William H. Robinson, Stanford University School of Medicine, Stanford, Calif. e-mail:wrobins@stanford.edu

2. Klein, R.J. et al. Science 308, 385-389 (2005)

3. Haines, J.L. et al. Science 308, 419-421 (2005)

4. Edwards, A.O. et al. Science 308, 421-424 (2005)

5. Rogers, J. et al. Res. Immunol. 143, 624-630 (1992)

6. Webster, S. et al. Neurobiol. Aging 18, 415-421 (1997)

7. Strohmeyer, R. et al. J. Neuroimmunol. 131, 135-146 (2002)

\section{COMPANIES AND INSTITUTIONS MENTIONED}

Alexion Pharmaceuticals Inc. (NASDAQ:ALXN), Cheshire, Conn. Brigham and Women's Hospital, Boston, Mass.

Case Western Reserve University School of Medicine, Cleveland, Ohio

CrystalGenomics Inc. (KOSDAQ:083790), Seoul, South Korea

Harvard Medical School, Boston, Mass.

Hospital for Special Surgery, New York, N.Y.

Novartis AG (NYSE:NVS; SIX:NOVN), Basel, Switzerland

Novartis Institutes for BioMedical Research, Basel, Switzerland

Noxxon Pharma AG, Berlin, Germany

Rush University Medical Center, Chicago, III.

Stanford University, Stanford, Calif.

Stanford University School of Medicine, Stanford, Calif.

University of Colorado Denver, Denver, Colo.

University of Padova, Padova, Italy

VA Palo Alto Health Care System, Palo Alto, Calif. 